\title{
Evaluation of cytology as secondary triage in visual inspection after application of $4 \%$ acetic acid-based cervical cancer screening program
}

\author{
Gauravi A. Mishra, Sharmila A. Pimple, Subhadra D. Gupta
}

\begin{abstract}
Context (Background): Visual-based screening techniques are low cost and have good sensitivity. Hence, they appear promising for primary screening in low-resource settings. However, to reduce referrals for diagnostic colposcopy, there is need to triage these screen-positive women with test with good specificity. Aims: The study aims to evaluate the performance of cytology as triage for visual inspection after application of $4 \%$ acetic acid (VIA) screen-positive women. Settings and Design: Community-based cervical cancer screening using VIA was implemented among socioeconomically disadvantaged women in Mumbai, India. Methods: Cytology was performed on screen-positive women. All primarily screen-positive women underwent colposcopy. Directed biopsies were obtained among women with positive findings on colposcopy. The gold standard used for final disease status was histopathology or negative colposcopy. Statistical Analysis Used:Test characteristics of cytology as triage test. Results: Among the 138,383 population, 16,424 eligible women were screened with VIA. 785 (4.78\%) women were VIA positive and 580 women participated in triage with cytology. The sensitivity and specificity of cytology at threshold of atypical squamous cells of undetermined significance in detecting $\geq$ cervical intraepithelial neoplasia (CIN) 2 were 75.0 and 94.7 , respectively. The positive and negative predictive values of cytology as triage test were 23.1 and 99.4 , respectively, and the false positivity and false negativity rates were 5.34 and 25.0, respectively. Conclusion: Cytology triage with VIA can reduce referrals for colposcopy to $4.97 \%$ of original referrals but may miss around $25 \%$, of high-grade CIN. The substantial reduction in referrals has special implication for low-resource settings, wherein compliance to referral and availability of diagnostic facilities are poor.
\end{abstract}

Key words: Cytology, screening, triage, visual inspection after application of $4 \%$ acetic acid

\section{Introduction}

Cervical cancer is the fourth most common cancer among women globally. India bears nearly one-fourth of the global cervical cancer burden. It is the second most common cancer among Indian women, being responsible for 132,314 new cases and 73,337 deaths annually. It accounts for $22.9 \%$ of all cancers among women in India. ${ }^{[1]}$ Here, population-based screening services are almost nonexistent because of the logistics challenges of implementing cytology-based screening program. Hence, alternative strategies are being explored. Visual-based screening techniques, i.e., visual inspection after application of $4 \%$ acetic acid (VIA) and visual inspection after application of Lugol's iodine are low cost and have fairly good sensitivity ${ }^{[2,3]}$ and thus appear promising for primary screening. The WHO has recommended visual-based techniques for primary screening of cervical cancers in countries with limited resources. ${ }^{[4,5]}$ However, the specificity of visual-based tests is low, resulting in high false-positive rates. ${ }^{[2]}$ Referral of all the screen positives (many of whom are false positives) for colposcopy will result in substantial costs for the health-care systems and may create unnecessary anxiety in the women and their families. ${ }^{[6]}$ Colposcopy services are still available only to a limited extent, with most of these facilities concentrated in the urban areas. Hence, there is need to triage the women screened positive on visual-based techniques with test with higher specificity to reduce referrals for colposcopy.

Cytology for cervical cancer screening has low sensitivity but fairly good specificity. ${ }^{[2,7]}$ It has reduced the incidence and mortality of cervical cancers in countries that implemented cytology-based screening over a period. ${ }^{[8-10]}$ There is also good evidence from lot of cross-sectional studies about its usefulness as a primary screening tool. ${ }^{[11]}$ However, for resource-poor countries, primary screening with cytology at population

\begin{tabular}{|c|}
\hline Access this article online \\
\hline Quick Response Code: \\
Q \\
Website: www.sajc.org \\
\hline DOI: $10.4103 /$ sajc.sajc_50_18 \\
\hline
\end{tabular}

Department of Preventive Oncology, Tata Memorial

Hospital, Mumbai, Maharashtra, India

Correspondence to: Dr. Gauravi A. Mishra,

E-mail:mishraga@tmc.gov.in level is still not feasible. Cytology can however be explored as a secondary triage test for women screened positive with visual-based tests. Only around one-fifth to one-tenth of the total eligible women will then have to undertake cytology. It will also limit the referrals for colposcopy as only women screened positive on primary and secondary screening tests will now have to be referred.

The present paper evaluates the test characteristics of cytology as a triage for VIA screen-positive women. The data for the present paper is from a service program on cervical cancer screening implemented in Mumbai, India.

\section{Methods}

A community-based cervical cancer screening project was implemented in selected slum clusters in Mumbai, India, during 2010-2017. Eleven slum clusters, not previously covered by any cancer screening program were selected. Meetings were held with the local leaders in order to ensure their support for the program. The project staff comprising mainly of the medical social workers (MSWs) and female primary health workers (PHWs) were recruited. The MSWs were graduates and the PHWs were $10^{\text {th }}$ grade educated. The MSWs received intensive training to conduct the project activities such as area mapping, conducting household surveys, obtaining informed consent, personal interviews of the eligible women, health education, counseling, and report preparation. PHWs were trained in insertion of speculum, preparation of $4 \%$ acetic acid, preparing the examination trolley, screening of cervical cancers by VIA, referral of screen-positive women, collection of cervical smears for cytology etc., Training to conduct VIA-based screening was offered to the PHWs using IARC manuals. ${ }^{[12]}$

The slum clusters were mapped to identify each and every lane and to identify every household. Surveys were conducted

This is an open access journal, and articles are distributed under the terms of the Creative Commons Attribution-NonCommercial-ShareAlike 4.0 License, which allows others to remix, tweak, and build upon the work non-commercially, as long as appropriate credit is given and the new creations are licensed under the identical terms.

For reprints contact: reprints@medknow.com 
in each slum cluster to enlist all the population residing in the slums, list total members of each household and the eligible women for screening. Women between the age group of 30 and 65 years residing in the selected slum clusters and with no history of cancer were considered as eligible for cervical cancer screening. Community-based screening clinics were set up with participation from the local community. The eligible women were invited to the nearby screening clinic, where they were explained the program and informed consent was obtained from women who were willing to participate. Their sociodemographic and risk factor information was recorded on a predesigned questionnaire after personal interviews. Women then participated in health education program explaining the risk factors, signs and symptoms, methods of early detection, and prevention of cervical cancers, after which they were invited to participate in cervical cancer screening. The PHWs conducted screening of cervix using VIA. All the screen-positive women and 10\% of the screen-negative women were independently reexamined by the medical officer at the camp place for quality assurance. The women screened negative were advised routine follow-up screening after 2 years at a nearby screening clinic. Cytology was offered to all VIA-positive women. The cervical smears were collected using small sterilized cotton swab stick. The smears were categorized as normal, inflammatory, atypical squamous cells of undetermined significance (ASCUS), low-grade squamous intraepithelial lesion (LSIL), high-grade squamous intraepithelial lesion, and invasive cancer at the cytology laboratory of Tata Memorial Hospital according to the Bethesda system. ${ }^{[13,14]}$

All primarily screen-positive women by VIA were referred to the nodal hospital for diagnostic Colposcopy by trained physicians. Cervical punch biopsy was performed for all women with positive findings on colposcopy. The histopathology reporting of the biopsy specimens was conducted at the hospital using the cervical intraepithelial neoplasia (CIN) system. ${ }^{[15]}$ The gold standard used for final disease status was histopathology report, whenever biopsy was performed or negative colposcopy. All women with cervical precancers and cancers received treatment at the nodal hospital according to the standard disease management guidelines. The project flowchart is represented in Figure 1.

The data was entered in IBM SPSS Statistics version 20 and then analyzed with STATA software version 10.0 (Stata Corp, College Station, TX, USA). The test characteristics of cytology as a secondary triage test, namely sensitivity, specificity, positive and negative predictive values, and false positive and false negative rates, were calculated with their $95 \%$ confidence intervals using $2 \times 2$ tables and standard formulae.

\section{Results}

The total population of the eleven slum clusters was 138,383. A total of 23,580 eligible women were enlisted from these clusters for cervical cancer screening, among which $21,422(90.85 \%)$ eligible women could be contacted during the camp period and $16,424(76.67 \%)$ women participated in cervical cancer screening by VIA at the screening camp. The sociodemographic profile of the participating women is as shown in Table 1.

Seven hundred and eighty-five (4.78\%) women were VIA positive and were offered cytology. Five hundred and South Asian Journal of Cancer • Volume 8 - Issue 2 • April-June 2019
Table 1: Distribution by important sociodemographic and risk factor variables among the participating women

\begin{tabular}{|c|c|}
\hline Variables & Participant women (\%) \\
\hline Total & 16,424 \\
\hline \multicolumn{2}{|l|}{ Age groups $(\%)$} \\
\hline $30-34$ & $3628(22.09)$ \\
\hline $35-39$ & $3681(22.41)$ \\
\hline $40-44$ & $3117(18.98)$ \\
\hline $45-49$ & $2641(16.08)$ \\
\hline $50-54$ & $1579(9.62)$ \\
\hline $55-59$ & $1065(6.48)$ \\
\hline $60-64$ & $713(4.34)$ \\
\hline \multicolumn{2}{|l|}{ Mean age (years) } \\
\hline Mean (SD) & $41.95(8.46)$ \\
\hline Range & $30-64$ \\
\hline \multicolumn{2}{|l|}{ Education (\%) } \\
\hline Literate without formal education & $965(5.88)$ \\
\hline Illiterate & $3573(21.75)$ \\
\hline School & $10,526(64.09)$ \\
\hline High school & $809(4.93)$ \\
\hline Sr. college & $378(2.30)$ \\
\hline Graduates and above & $173(1.05)$ \\
\hline \multicolumn{2}{|l|}{ Monthly family income (Rs.) (\%) } \\
\hline$<2000$ & $188(1.14)$ \\
\hline $2001-5000$ & $5763(35.09)$ \\
\hline $5001-10,000$ & $9633(58.65)$ \\
\hline $10,001-15,000$ & $675(4.11)$ \\
\hline Above 15,000 & $165(1.01)$ \\
\hline \multicolumn{2}{|l|}{ Occupation (\%) } \\
\hline Homemaker & $13,105(79.79)$ \\
\hline Manual labor & $2224(13.54)$ \\
\hline Service (White Collar) & $656(3.99)$ \\
\hline Self-employed & $438(2.67)$ \\
\hline Others & $1(0.01)$ \\
\hline \multicolumn{2}{|l|}{ Religion (\%) } \\
\hline Hindu & $12,858(78.29)$ \\
\hline Muslim & $1902(11.58)$ \\
\hline Others & $1664(10.13)$ \\
\hline \multicolumn{2}{|l|}{ Language (\%) } \\
\hline Marathi & $12,094(73.64)$ \\
\hline Hindi & $2986(18.18)$ \\
\hline Others & $1344(8.18)$ \\
\hline \multicolumn{2}{|l|}{ Marital status (\%) } \\
\hline Unmarried & $6(0.04)$ \\
\hline Married & $14,405(87.71)$ \\
\hline Divorced & $58(0.35)$ \\
\hline Widowed & $1781(10.84)$ \\
\hline Separated & $174(1.06)$ \\
\hline \multicolumn{2}{|l|}{ Menstrual status (\%) } \\
\hline Premenopausal & $10,792(65.71)$ \\
\hline Perimenopausal & $1173(7.14)$ \\
\hline Postmenopausal & $4459(27.15)$ \\
\hline \multicolumn{2}{|l|}{ Mean age at menarche (years) } \\
\hline$n$ & 16,424 \\
\hline Range & $10-22$ \\
\hline Mean (SD) & $13.84(1.04)$ \\
\hline \multicolumn{2}{|l|}{ Mean age at menopause (years) } \\
\hline$n$ & 4459 \\
\hline Range & $16-56$ \\
\hline Mean (SD) & $44.68(3.92)$ \\
\hline
\end{tabular}


Table 1: Contd...

\begin{tabular}{lc}
\hline Variables & Participant women (\%) \\
\hline Mean age at marriage (years) & 16,418 \\
$n$ & $5-42$ \\
Range & $18.63(3.98)$ \\
Mean (SD) & \\
Mean age at first childbirth (years) & 16,007 \\
$n$ & $12-43$ \\
Range & $20.94(3.75)$ \\
Mean (SD) & \\
Average number of children & 15,867 \\
$n$ & $1-13$ \\
Range & $2.83(1.30)$ \\
Mean (SD) & \\
Tobacco use (\%) & $7558(46.02)$ \\
Yes & $8866(53.98)$ \\
No & \\
History of cancer screening (\%) & $226(1.38)$ \\
Yes & $16,198(98.62)$ \\
No
\end{tabular}

SD: Standard deviation

eighty screen-positive women complied with cytology (conventional PAP smears) and 582 screen-positive women complied to diagnostic colposcopy. Figure 2 shows the outcome of the screening program.

In six cases, the cytology was reported as inadequate and were excluded from the analysis. Conventional cytology was reported as normal in 535 women, ASCUS in 25 women, Low Grade Squamous Intraepithelial Lesion (LGSIL) in 6 women, and High Grade Squamous Intraepithelial Lesion (HGSIL) in 8 women. No case of carcinoma was reported on cytology. On histopathological examination of the cervical biopsy samples, 507 were reported as benign, 14 atypia/HPV changes, 49 CIN 1, 6 CIN 2, and 6 CIN 3. No case of cervical cancer was reported on histopathology. Table 2 shows the agreement between cytology and histopathology reports.

Final disease status by screening test result for the women positive on VIA and who had simultaneously undergone cytology, at the threshold of ASCUS and LSIL is as shown in Table 3.

Table 4 shows the accuracy estimates for different disease status for the women found positive on VIA and who had simultaneously undergone cytology for the outcome $\geq$ CIN 1 and $\geq$ CIN 2, using ASCUS and LSIL as threshold. The sensitivity of cytology as a triage test using ASCUS and LSIL as thresholds for the outcomes $\geq$ CIN 1 is 25.9 and 13.8 and for $\geq$ CIN 2 is 75.0 and 50.0, respectively. The specificity of cytology as a triage test using ASCUS and LSIL as threshold for the outcomes $\geq$ CIN 1 is 95.3 and 98.8 and for the outcome $\geq$ CIN 2 is 94.7 and 98.6, respectively. The positive and negative predictive values of cytology as triage test with ASCUS as threshold for the outcomes $\geq$ CIN 1 are 38.5 and 92.0 and for the outcomes $\geq$ CIN 2 are 23.1 and 99.4, respectively. The same with LSIL as threshold for the outcomes $\geq$ CIN 1 and CIN 2 are 57.1 and 91.1 and 42.9 and 98.9, respectively. The false-positive and false-negative rates of cytology as triage test are 4.65 and 74.14 and 5.34 and 25.0 with ASCUS as threshold for the outcomes $\geq$ CIN 1 and $\geq$ CIN 2, while with LSIL as threshold, they are 1.16 and 86.21 and 1.42 and 50.0, respectively.

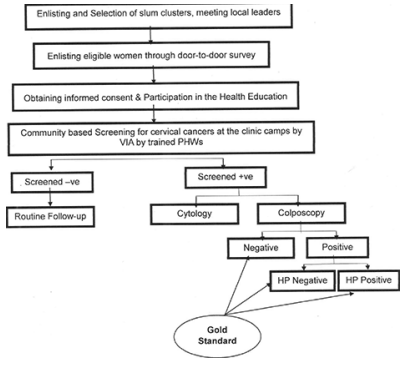

Figure 1: Project schema

\section{Discussion}

A triage test is a test that is applied to primarily screen-positive individuals, before a diagnostic test, to further shortlist the probably diseased individuals. Most low-resource settings may not have easily accessible health-care facilities providing colposcopy for all primary screen-positive women. A triage test, in such settings, reduces the number of women that need to get back for repeat testing. An ideal cervical cancer screening strategy using triage test should be able to identify women needing colposcopic referral and separate the rest of the women who are at low risk of cervical cancer. ${ }^{[16]}$

In a cancer screening project, there is a loss of participation of women at many levels during the program. In the present program, $76.67 \%$ women participated in VIA-based cervical cancer screening conducted in local community-based camps. In an earlier community-based cervical cancer screening study in Mumbai, $71.5 \%$ women participated in the first round. ${ }^{[17]}$

Among those who participated in the present program, 79.56\% were below 50 years, $21.75 \%$ were illiterates, $93.74 \%$ had monthly family income between Indian rupee 2000/- and 10,000/-, 79.79\% were homemakers, $78.29 \%$ belonged to Hindu religion, $73.64 \%$ were Marathi speaking, $87.71 \%$ were married, $65.71 \%$ were premenopausal, $46.02 \%$ used tobacco, and $1.38 \%$ had undertaken cancer screening in the past. The mean age at menarche, menopause, marriage, and first childbirth were 13.84, 44.68, 18.63, and 20.94, respectively, and the average number of children born was 2.83 .

In the present program, $74 \%$ of the VIA-positive women complied for cytology and colposcopy. The compliance of VIA positives to referral was $68 \%$ in the Kenyan study. ${ }^{[18]}$ Of the 574 VIA-positive women who complied to cytology and had adequate cells in the present program, 39 women had findings ASCUS and above on cytology and 58 women had findings CIN 1 and above on histopathology.

In the present study, using positive histology or negative colposcopy as the gold standard, the sensitivity of cytology triage is extremely poor for the outcome CIN 1, at the threshold of LSIL (13.8). It remains poor even with the threshold of ASCUS (25.9). The sensitivity of cytology as triage test is best for outcome $\geq$ CIN 2 with ASCUS threshold (75.0) followed by LSIL as threshold (50.0). In a study from Kenya, the sensitivity of cervical cytology as a triage test on VIA-positive women was $62 \%$ and $80 \%$ with CIN $1+$ and CIN $2+$ as disease outcomes, respectively. ${ }^{[18]}$ In one of the Indian studies, the sensitivity of sequential testing of VIA followed by PAP was 75.8. ${ }^{[19]}$ An earlier Mumbai study reported sensitivity of $67.9 \%, 62.3 \%$, and $57.4 \%$ of cytology at the threshold of ASCUS+, LGSIL+, and HGSIL, respectively, with CIN 2+ histopathology as the gold standard on all VIA-positive women. ${ }^{[20]}$

South Asian Journal of Cancer • Volume 8 •Issue 2 • April-June 2019 
Table 2: Agreement between cytology and colposcopy-directed biopsy

\begin{tabular}{lcccccc}
\hline Histopathology & \multicolumn{5}{c}{ Cytology } \\
\cline { 2 - 7 } & Benign & HPV & ASCUS & LGSIL & HGSIL & Invasive cancer \\
\hline Benign & 478 & 0 & 18 & 5 & 0 & 0 \\
Atypia or HPV & 14 & 0 & 0 & 0 & 0 & 0 \\
CIN 1 & 40 & 0 & 4 & 1 & 1 & 0 \\
CIN 2 & 3 & 0 & 2 & 0 & 1 & 0 \\
CIN 3 & 0 & 0 & 1 & 0 & 5 & 0 \\
Invasive cancer & 0 & 0 & 0 & 0 & 0 & 0 \\
Total & 535 & 0 & 25 & 6 & 8 & 0 \\
\hline
\end{tabular}

ASCUS: Atypical cells of undetermined significance, CIN: Cervical intraepithelial neoplasia, HPV: Human papillomavirus, LGSIL: Low grade squamous intraepithelial lesion, HGSIL: High grade squamous intraepithelial lesion

Table 3: Final disease status by screening test result for the women found positive on visual inspection with acetic acid and who had simultaneously undergone cytology

\begin{tabular}{|c|c|c|c|c|c|c|}
\hline \multirow[t]{3}{*}{ Final diagnosis } & \multicolumn{6}{|c|}{ Cytology } \\
\hline & \multicolumn{3}{|c|}{ Using ASCUS threshold } & \multicolumn{3}{|c|}{ Using LSIL threshold } \\
\hline & Negative & Positive & Total & Negative & Positive & Total \\
\hline Normal & 492 & 24 & 516 & 510 & 6 & 516 \\
\hline CIN 1 & 40 & 6 & 46 & 44 & 2 & 46 \\
\hline CIN 2 & 3 & 3 & 6 & 5 & 1 & 6 \\
\hline CIN 3 & 0 & 6 & 6 & 1 & 5 & 6 \\
\hline Total & 535 & 39 & 574 & 560 & 14 & 574 \\
\hline
\end{tabular}

ASCUS: Atypical cells of undetermined significance, CIN: Cervical intraepithelial neoplasia, LSIL: Low-grade squamous intraepithelial lesion

Table 4: Test characteristics of visual inspection with acetic acid as primary screening test and cytology as a triage test, using positive histology or negative colposcopy as the gold standard

\begin{tabular}{|c|c|c|c|c|c|c|c|c|}
\hline \multirow[t]{4}{*}{ Parameter } & \multicolumn{8}{|c|}{ Cytology as triage } \\
\hline & \multicolumn{4}{|c|}{ Disease status $\geq$ CIN 1} & \multicolumn{4}{|c|}{ Disease status $\geq$ CIN 2} \\
\hline & \multicolumn{2}{|c|}{$\geq$ ASCUS } & \multicolumn{2}{|c|}{$\geq$ LSIL } & \multicolumn{2}{|c|}{$\geq$ ASCUS } & \multicolumn{2}{|c|}{$\geq$ LSIL } \\
\hline & Percentage & $95 \% \mathrm{CI}$ & Percentage & $95 \% \mathrm{CI}$ & Percentage & $95 \% \mathrm{CI}$ & Percentage & $95 \% \mathrm{CI}$ \\
\hline Sensitivity & 25.9 & $15.3-39$ & 13.8 & $6.15-25.4$ & 75.0 & $42.8-94.5$ & 50.0 & $21.1-78.9$ \\
\hline Specificity & 95.3 & $93.2-97$ & 98.8 & $97.5-99.6$ & 94.7 & $92.5-96.4$ & 98.6 & $97.2-99.4$ \\
\hline PPV & 38.5 & $23.4-55.4$ & 57.1 & $28.9-82.3$ & 23.1 & $11.1-39.3$ & 42.9 & $17.7-71.1$ \\
\hline NPV & 92.0 & $89.3-94.1$ & 91.1 & $88.4-93.3$ & 99.4 & 98.4-99.9 & 98.9 & $97.7-99.6$ \\
\hline FPR & 4.65 & & 1.16 & & 5.34 & & 1.42 & \\
\hline FNR & 74.14 & & 86.21 & & 25.0 & & 50.0 & \\
\hline
\end{tabular}

ASCUS: Atypical cells of undetermined significance, CIN: Cervical intraepithelial neoplasia, LSIL: Low-grade squamous intraepithelial lesions, NPV: Negative predictive value, PPV: Positive predictive value, FPR: False-positive rates, FNR: False-negative rates, CI: Confidence interval

In the present study, the specificity of cytology as a triage test was best with LSIL threshold both for the outcomes $\geq$ CIN 1 (98.8) and $\geq$ CIN 2 (98.6), while it was comparatively lesser using ASCUS threshold both for the outcomes $\geq$ CIN 1 (95.3) and $\geq$ CIN 2 (94.7). In the African study from Kenya, the specificity of cervical cytology as a triage test on VIA-positive women was poor for CIN $2+$ outcome $(48 \%)$ and for CIN $1+$ outcome $(50 \%) \cdot{ }^{[18]}$ The specificity of cytology as a triage test was 83.0 in an Indian study ${ }^{[19]}$ In the Mumbai study, the specificity of pap as triage test was $99.4 \%, 98.0 \%$, and $96.8 \%$ to detect HGSIL+, LGSIL+, and ASCUS+ with histopathology CIN $2+$ as the gold standard on VIA-positive women. ${ }^{[20]}$

The PPV of cytology as triage test is best with LSIL as threshold for the outcome $\geq$ CIN 1 (57.1) and $\geq$ CIN 2 (42.9). It is much lesser with ASCUS threshold both for outcome $\geq$ CIN 1 (38.5) and outcome $\geq$ CIN 2 (23.1). The negative predictive value of cytology as triage is best for outcome $\geq$ CIN 2 (99.4) with ASCUS threshold, followed by LSIL threshold (98.9). For the outcome $\geq$ CIN 1, the NPV is much lesser (92.0) for ASCUS threshold and LSIL threshold (91.1). The PPV and NPV of cytology triage in the Kenyan study were $36 \%$ and $75 \%$, respectively, ${ }^{[18]}$ and in the Indian study were 50 and 93.9 , respectively. ${ }^{[19]}$ The PPV and South Asian Journal of Cancer Volume 8 •Issue 2 • April-June 2019
NPV of cytology triage in the Mumbai cross-sectional study were $89.4 \%$ and $96.2 \%$, respectively, for HGSIL to detect CIN2+ lesions. ${ }^{[20]}$ The PPV and NPV of cytology in the African study were $12 \%$ and $97 \%$, respectively. ${ }^{[18]}$

The huge referral burden of false-positive cases resulting from low specificity is the major limitation of VIA-based screening. ${ }^{[21]}$ When cytology was used as triage test in the present study, the referral burden was reduced from 785 to 39 , i.e., only $4.97 \%$ of the original referrals. However, even after combining all VIA-positive cases with cytology, some cases still remained false positives. The false-positive rate of cytology triage was much lesser with LSIL as threshold for the outcomes $\geq$ CIN 1 (1.16) and outcomes $\geq$ CIN 2 (1.42) as compared to ASCUS as threshold for the outcomes $\geq$ CIN 1 (4.65) and $\geq \operatorname{CIN} 2$ (5.34), respectively. It is important to limit the number of false positives in a screening program, since these women may undergo lot of psychological distress associated with long-term sequelae of unnecessary investigations and overtreatment. ${ }^{[22]}$

The major negative aspect of triaging is missing of the true cases or the false negativity. In the present study, the false-negative rates of cytology triage were 74.14 and 25.0 with ASCUS as threshold and 86.21 and 50.0 with LSIL as 
threshold, for the outcomes $\geq$ CIN 1 and $\geq$ CIN 2, respectively. The Mumbai study had a false-negative rate of $42.6 \%$ cytology triage. ${ }^{[20]}$

Compliance to diagnostic referral of screen-positive women may remain poor in many low-resource settings, wherein the diagnostic centers may be situated at far off distances and there may be other sociocultural and logistic barriers for good compliance. Hence, if the screen positives also receive a triage test at the community level, the number of women needed to be referred to higher centers will reduce, without loss of diagnostic accuracy. Further, there is a loss of compliance at the level of diagnosed cases complying with the treatment. All this can be prevented if treatment is also provided in the same sitting, at the community level. This can be achieved by the use of "see and treat" strategy. However, if all VIA-positive women are to be treated, it will lead to overtreatment, since VIA has a high false-positive rate and many women will get unnecessarily treated. However, overtreatment will be several times less if only VIA and cytology positive women are treated with "see and treat" strategy in the subsequent visit.

The limitation of cytology triage is that it requires laboratory services and skilled cytologists and also does not provide immediate results. Hence, it necessitates revisits by patients for further management, which remains a challenge for implementing cervical cancer screening program in low-resource settings. Meticulous quality control practices for cytology need to be maintained even when it is serving as a triage test.

\section{Conclusion}

To conclude, the test characteristics of cytology triage are much better with CIN $2+$ as the gold standard compared to CIN 1+. Cytology triage of VIA-positive cases reduces the referral burden to only $5 \%$ of the original referrals. However, this also leads to missing of some precancer cases. The loss is much lesser $(25 \%)$ with CIN $2+$ as the gold standard and ASCUS+ as cutoff. The study findings have special implication for low-resource settings, wherein compliance to referral and availability of diagnostic facilities are poor.

\section{Acknowledgment}

We gratefully acknowledge the funding support for the present service program through the Department of Atomic Energy (DAE) under the XIth 5-year plan period. The patient care cost is partly subsidized by the Tata Memorial Hospital and rest is supported by the DAE and the Women's Cancer Initiative (WCI). A mobile vehicle for screening was donated to the project by the WCI. The sponsors (DAE and WCI) had no role in planning, designing, or conducting the program. The authors wish to acknowledge the contributions of the following persons who have been directly or indirectly associated with the program, namely, Pranoti Satpute, Sheetal Kulkarni, Farhat Chaudhary, Heena Shaikh, Meenal Rokade, Vasundhara Kulkarni, Padma Sadalge, Nabha Kadam, Neha Malaiya, Pallavi Uplap, Yogesh Kumar, Srikant Atreya, Nilesh Ingole, Kavita Anand, Parishi Majmudar, Rajendra Kerkar, Amita Maheshwari, Rajendra Badwe, Vani Parmar, Neeta Nair, Meenakshi Thakur, Subhash Ramani, Shubhada Kane, Ajit Dulhan, Shylasree, and all the staff working for this project.

Financial support and sponsorship

The study was supported by the Department of Atomic Energy (DAE), Government of India, under the $\mathrm{XI}^{\text {th }} 5$-year plan. Tata Memorial Hospital partly subsidized the treatment cost for the patients and part of it was supported by the Women's Cancer Initiative (WCI) and the DAE. The WCI also donated a mobile screening vehicle for the program.

\section{Conflicts of interest}

There are no conflicts of interest.

\section{References}

1. Ferlay J, Soerjomataram I, Ervik M, Dikshit R, Eser S, Mathers C, et al. GLOBOCAN 2012 v1.0, Cancer Incidence and Mortality Worldwide: IARC Cancer Base No 11. Lyon, France: International Agency for Research on Cancer; 2013. Available from: http://www.globocan.iarc. fr. [Last accessed on 2017 May 17].

2. Arbyn M, Sankaranarayanan R, Muwonge R, Keita N, Dolo A, Mbalawa CG, et al. Pooled analysis of the accuracy of five cervical cancer screening tests assessed in eleven studies in Africa and India. Int J Cancer 2008; 123:153-60.

3. Sauvaget C, Fayette JM, Muwonge R, Wesley R, Sankaranarayanan R. Accuracy of visual inspection with acetic acid for cervical cancer screening. Int J Gynaecol Obstet 2011;113:14-24.

4. WHO. Guidelines for Screening and Treatment of Precancerous Lesions for Cervical Cancer Prevention. Geneva: World Health Organization; 2013.

5. Guidelines for Cervical Cancer Screening Programme. National Cancer Control Programme. GOI-WHO Collaborative Programme. Chandigarh, India: PGI; 2006

6. Pimple S, Muwonge R, Amin G, Goswami S, Sankaranarayanan R, Shastri SS, et al. Cytology versus HPV testing for the detection of high-grade cervical lesions in women found positive on visual inspection in Mumbai, India. Int J Gynaecol Obstet 2010;108:236-9.

7. Nanda K, McCrory DC, Myers ER, Bastian LA, Hasselblad V, Hickey JD, et al. Accuracy of the papanicolaou test in screening for and follow-up of cervical cytologic abnormalities: A systematic review. Ann Intern Med 2000; 132:810-9.

8. Herbert A, Stein K, Bryant TN, Breen C, Old P. Relation between the incidence of invasive cervical cancer and the screening interval: Is a five year interval too long? J Med Screen 1996;3:140-5.

9. Arbyn M, Raifu AO, Weiderpass E, Bray F, Anttila A. Trends of cervical cancer mortality in the member states of the European Union. Eur J Cancer 2009;45:2640-8

10. Lăără E, Day NE, Hakama M. Trends in mortality from cervical cancer in the Nordic countries: Association with organised screening programmes. Lancet 1987; 1:1247-9.

11. Peirson L, Fitzpatrick-Lewis D, Ciliska D, Warren R. Screening for cervical cancer: A systematic review and meta-analysis. Syst Rev 2013;2:35.

12. Sankaranarayanan R, Wesley R, editors. A Practical Manual on Visual Screening for Cervical Neoplasia. IARC Technical Publication; No. 41. Lyon, France: International Agency for Research on Cancer Press; 2003.

13. Solomon D, Davey D, Kurman R, Moriarty A, O'Connor D, Prey M, et al. The 2001 Bethesda system: Terminology for reporting results of cervical cytology. JAMA 2002;287:2114-9.

14. Nayar R, Wilbur DC. The pap test and Bethesda 2014. "The reports of my demise have been greatly exaggerated." (after a quotation from Mark Twain). Acta Cytol 2015;59:121-32.

15. Darragh TM, Colgan TJ, Thomas Cox J, Heller DS, Henry MR, Luff RD, et al. The lower anogenital squamous terminology standardization project for HPV-associated lesions: Background and consensus recommendations from the College of American Pathologists and the American Society for Colposcopy and Cervical Pathology. Int J Gynecol Pathol 2013;32:76-115.

16. Wentzensen N, Schiffman M, Palmer T, Arbyn M. Triage of HPV positive women in cervical cancer screening. J Clin Virol 2016;76 Suppl 1:S49-55.

17. Shastri SS, Mittra I, Mishra GA, Gupta S, Dikshit R, Singh S, et al. Effect of VIA screening by primary health workers: Randomized controlled study in Mumbai, India. J Natl Cancer Inst 2014;106:dju009.

18. Lewis KC, Tsu VD, Dawa A, Kidula NA, Chami IN, Sellors JW, et al. A comparison of triage methods for Kenyan women who screen positive for cervical intraepithelial neoplasia by visual inspection of the cervix with acetic acid. Afr Health Sci 2011;11:362-9.

19. Bhatla N, Puri K, Kriplani A, lyer VK, Mathur SR, Mani K, et al. Adjunctive testing for cervical cancer screening in low resource settings. Aust $\mathrm{N}$ Z J Obstet Gynaecol 2012;52:133-9.

South Asian Journal of Cancer • Volume 8 •Issue 2 • April-June 2019 
20. Pimple SA, Amin G, Goswami S, Shastri SS. Evaluation of colposcopy vs. cytology as secondary test to triage women found positive on visual inspection test. Indian J Cancer 2010;47:308-13.

21. Sankaranarayanan R, Gaffikin L, Jacob M, Sellors J, Robles S. A critical assessment of screening methods for cervical neoplasia. Int J Gynaecol Obstet 2005;89 Suppl 2:S4-12.

22. Sankaranarayanan R. Screening for cancer in low- and middle-income countries. Ann Glob Health 2014;80:412-7.ssssss 